\title{
Development and Validation of RP-HPLC Method for the Simultaneous Estimation of Atenolol and Amlodipine in Tablet Dosage Form
}

\author{
Md. Ahsanul Haque ${ }^{1}$, Asma Naznin ${ }^{1}$, A. N.M Hamidul Kabir ${ }^{2}$, \\ Md. Khalid Hossain ${ }^{3}$ and S. M. Ashraful Islam ${ }^{1}$ \\ ${ }^{1}$ Department of Pharmacy, The University of Asia Pacific, Dhanmondi, Dhaka-1209, Bangladesh \\ ${ }^{2}$ Department of Applied Chemistry \& Chemical Engineering, Faculty of Engineering, University of Dhaka, \\ Dhaka-1000, Bangladesh \\ ${ }^{3}$ Department of Pharmaceutical Chemistry, Faculty of Pharmacy, University of Dhaka, \\ Dhaka-1000, Bangladesh
}

\begin{abstract}
A simple, selective and rapid reversed phase high performance liquid chromatographic (RPHPLC) method for the analysis of atenolol and amlodipine in tablet has been developed and validated. The chromatographic system consisted of a LC-20 AT pump, SPD-20 A UV/visible detector. The separation was achieved from C18 column at ambient temperature with a mobile phase consisting of methanol-acetonitrile buffer (solution of ammonium acetate and sodium pentanesulphonate ratio, 55:10:35 v/v, $\mathrm{P}^{\mathrm{H}}=3.00$ adjusted with phosphoric acid) at a flow rate of $1 \mathrm{ml} / \mathrm{min}$ and the retention time was about 1.67 minutes for atenolol and 5.0 minutes for amlodipine. The method is selective and able to resolve drug peaks from formulation excipients. The peaks of atenolol and amlodipine were well separated (resolution 11.65). The calibration curves were linear over the concentration range of $80 \%$ to $120 \%$ ( $r^{2}=0.999$ for both the drugs). The proposed method is accurate with $100.72 \%$ recovery for atenolol and $99.44 \%$ recovery for amlodipine and precise (\%RSD of intra day variation were $0.53-0.152$ for atenolol and 0.067-1.518 for amlodipine and \%RSD of inter day variation were 0.024-1.518 for atenolol and 0.034-1.518 for amlodipine). The method has been used to test market products (six brands) and potency was found within limit (99.02\%- 100.02\% for atenolol and $97.4 \%-100.4 \%$ for amlodipine). Therefore, this method can be used as a more convenient and efficient option for the analysis of atenolol and amlodipine in tablet dosage form.
\end{abstract}

Key words: Atenolol, amlodipine, method validation, HPLC, quantitative analysis

\section{INTRODUCTION}

Atenolol (Figure 1) chemically 2-[4-[(2 RS)-2hydroxy-3-(1methylethyl)amino] propoxy] phenyl] acetamide, is a $\beta$-blocker seem to be equally effective as an antihypertensive, anti-anginal and antiarrhymthmic drug. It is widely used as cardiovascular drug in combination with amlodipine. ${ }^{1}$

Correspondence to:

S.M. Ashraful Islam

Tel: 880-2-8629367, Extn. 136

E-mail: ashraf@uap-bd.edu

Dhaka Univ. J. Pharm. Sci. 9(2): 131-138, 2010 (December)<smiles>CCCCCCC(C)NC[C@H](O)COc1ccc(CC(N)=O)cc1</smiles>

Figure 1: Structure of atenolol

Amlodipine besylate [Figure 2] chemically 3-Ethyl 5-methyl (4RS)-2-[(2-aminoethoxy) methyl]4-(2-chlorophenyl)-6-methyl-1,4-dihydropyridine3,5-dicarboxylate benzenesulphonate is a long-acting calcium channel blocker used for hypertension and angina pectoris. ${ }^{2,3}$ 


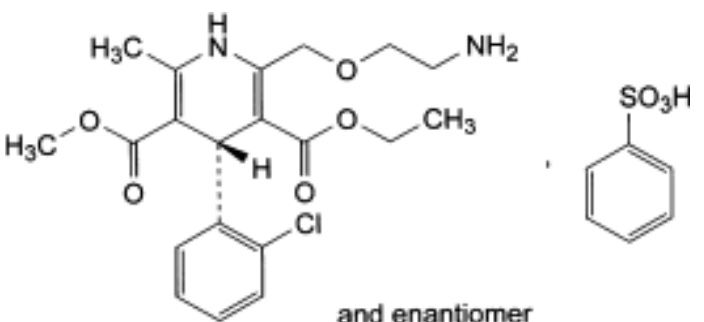

Figure 2. Structure of amlodipine besylate.

Combination of atenolol and amlodipine can simplify dosing regimens, improve compliance, improve hypertension control, decrease dosedependent side effects and reduce cost as the firstline treatment of hypertension. ${ }^{4}$ Li-Ping et al., 2005 demonstrated that the combination of atenolol and amlodipine is synergistic in lowering and stabilizing $\mathrm{BP}$ and this synergism is highest when the dose proportion of the two drugs is $10: 1 .^{5}$

The official method for estimation of amlodipine includes non-aqueous titration ${ }^{6} \& \mathrm{HPLC}^{7}$ and for atenolol is HPLC. ${ }^{8,9}$ Literature survey also revealed that various analytical methods have been reported for the assay of atenolol ${ }^{10}$ and amlodipine ${ }^{11-14}$ in individual formulations and combined with other drugs. $^{15,18}$ Simultaneous estimation of atenolol and amlodipine by spectroscopic ${ }^{19}$, RP-HPLC ${ }^{20,21}$ and HP-TLC ${ }^{22}$ are also available.

Various excipients present in formulations may interfere the determination of atenolol and amlodipine by spectroscopic method. On the other hand HP-TLC is not that accurate for quantitative estimation and the HPLC methods are not free from limitations. The British Pharmacopoeia describes UV method for the assay of atenolol tablet and HPLC methods for atenolol syrup. United States Pharmacopeia describes HPLC method for the determination of atenolol tablet. But analysis of tablet containing atenolol and amlodipine has not been reported in British Pharmacopoeia or in United States Pharmacopeia. So, the present work was undertaken with the aim to develop and validate an economic, rapid reversed-phase high performance liquid chromatographic method with high resolution according to ICH guideline. We used sodium pentanesulphonate along with ammonium acetate to prepare buffer solution which was mixed with methanol and acetonitrile (35:55:10 v/v). So far as we know this combination and ratio of mobile phase has not been reported in any literature. Furthermore analyses of six commercial brands of atenolol and amlodipine tablets were also included in this study.

\section{MATERIALS AND METHODS}

Material. The amlodipine besylate and atenolol were provided by Renata Ltd. Dhaka, Bangladesh. Acetonitrile and methanol were of HPLC grade and were purchased from E. Merck, Darmstadt, Germany. Ammonium acetate, sodium pentanesulphonate, orthophosphoric acid and other reagents were of analytical-reagent grade and purchased from E. Merck, Darmstadt, Germany. Water was deionised and double distilled. Six commercial brands of tablets containing $50 \mathrm{mg}$ atenolol and $5 \mathrm{mg}$ amlodipine (as amlodipine besylate) were purchased from local drug shops in Dhaka city after checking their manufacturing license numbers, batch numbers, production and expiry dates. They were randomly coded as T-1 to T-6 and stored properly.

Instrumentation. A Shimadzu (Japan) HPLC system consisting of a CMB-20 Alite system controller, two LC-20AT pumps, SIL-20A autosampler and CTO-10ASVP column oven were used. Ultraviolet detection was achieved with a SPD-20A UV-VIS detector (Shimadzu, Japan). The drug analysis data were acquired and processed using LC solution (Version 1.2, Shimadzu, Japan) software running under Windows XP on a Pentium PC.

Chromatographic conditions. The mobile phase, a mixture of buffer, methanol and acetonitrile (35:55:10 v/v) pumped at a flow rate of $1.0 \mathrm{ml} / \mathrm{min}$ through the column (C18; 5 $\mu$, 4.6 X 150 mm, Waters, USA) at ambient temperature. The mobile phase was degassed prior to use under vacuum by filtration through a $0.2 \mu$ nylon membrane. Concentrations were measured at $237 \mathrm{~nm}$ by UV detector at a sensitivity of 0.0001 .

Preparation of standard solution. For $100 \%$ standard solution of target concentration $50 \mathrm{mg}$ 
atenolol and $5 \mathrm{mg}$ amlodipine (as amlodipine besylate) were weighed, dissolved and sonicated in mobile phase to produce $100 \mathrm{ml}$. $80 \%, 90 \%, 110 \%$, and $120 \%$ standard solutions of target concentration were also prepared in the same way.

Preparation of sample solution. 20 tablets were accurately weighed and the average weight was calculated. The tablets were grinded to a fine powder with the help of mortar and pestle. Then, the amount of powder equivalent to average weight of a tablet was transferred to a volumetric flask, dissolved in mobile phase and shaken for about 10 minutes then filtered through filter paper. The filtered solution was further diluted in the mobile phase to make the final concentration of working sample equivalent to $100 \%$ of target concentration.

Development and validation of HPLC method. Present study was conducted to obtain a new, affordable, cost-effective and convenient method for HPLC determination of atenolol and amlodipine in tablet dosage form. The experiment was carried out according to the official specifications of USP-30, ICH- 1996, Global Quality Guidelines-2002. ${ }^{8,23-24}$ The method was validated for the parameters like system suitability, selectivity, linearity, accuracy, precision, and robustness.

System suitability. The system suitability was assessed by six replicate analysis of atenolol and amlodipine at a $100 \%$ level to verify the resolution and reproducibility of the chromatographic system adequate for the analysis to be done. This method was evaluated by analyzing the repeatability of retention time, peak area for both atenolol and amlodipine tailing factor, theoretical plates (Tangent) of the column and resolution between the peaks of atenolol and amlodipine.

Selectivity. Selectivity was determined in the presence of common excipients used in the tablet formulation. Sample containing 100\% atenolol and amlodipine was injected first. Then the samples mixed with three different placebo formulations were injected to find out the selectivity of the method.
Linearity. Linearity of the method was determined by constructing calibration curves. Standard solutions of atenolol and amlodipine at different concentrations level (80\%, 90\%, 100\%, $110 \%$, and $120 \%$ ) were used for this purpose. Before injection of the solutions, the column was equilibrated for at least $30 \mathrm{~min}$ with the mobile phase. Each measurement was carried out in six replicates to verify the reproducibility of the detector response at each concentration level. The peak areas of the chromatograms were plotted against the concentrations of atenolol and amlodipine to obtain the calibration curves. The five concentrations of the standard were subjected to regression analysis to calculate calibration equation and correlation coefficients.

Accuracy. The accuracy is the closeness of agreement between the true value and test result. Accuracy was determined by means of recovery experiments, by addition of active drug to placebo formulations. The accuracy was calculated from the test results as the percentage of the analyte recovered by the assay.

Precision. The precision of the method was determined by repeatability (intra-day) and intermediate precision (inter-day) study. Repeatability was determined by performing four repeated analysis of the three standard solutions (90\%, 100\% and $110 \%$ of target concentration) of atenolol and amlodipine on the same day, under the same experimental conditions. The intermediate precision of the method was assessed by carrying out the analysis of previous standard solutions on three different days (inter-day) in the same laboratory. The relative standard deviation (\% RSD) was determined in order to assess the precision of the method.

Robustness. The robustness of the method was assessed by altering the some experimental conditions such as, by changing the flow rate from 0.9 to $1.1 \mathrm{ml} / \mathrm{min}$, amount of acetonitrile $(10 \%$ to $15 \%$,) the temperature of the column $\left(28^{\circ} \mathrm{C}\right.$ to $\left.32{ }^{\circ} \mathrm{C}\right)$ and $\mathrm{P}^{\mathrm{H}}$ of the mobile phase. 


\section{RESULTS AND DISCUSSION}

System suitability. The system suitability tests were carried out to evaluate the resolution and reproducibility of the system for the analysis. Table 1 summarized the test results of system suitability study. All the chromatograms showed the same retention time for atenolol (1.676 min with \% RSD 0.084 ) and amlodipine (5.004 min with \%RSD 0.39) from the six consecutive injections of the standard solution which indicates a good system for analysis. The mean theoretical plate count, based on USP tangent calculations was 3078.3 for atenolol and 2984.31for amlodipine and the resolution between atenolol and amlodipine was 11.65

Table 1. Result of system suitability tests of atenolol and amlodipine

Name of drug-atenolol

\begin{tabular}{ccccc}
\hline Injection No. & Retention time & Area & Theoretical plates & Tailing factor \\
\hline 1 & 1.677 & 3686546.00 & 3078.025 & 1.406 \\
2 & 1.675 & 3687323.00 & 3077.843 & 1.405 \\
3 & 1.676 & 3685214.00 & 3078.468 & 1.406 \\
4 & 1.678 & 3685944.00 & 3079.15 & 1.406 \\
5 & 1.674 & 3686674.00 & 3077.843 & 1.405 \\
6 & 1.676 & 3687404.00 & 3078.468 & 1.406 \\
Average & 1.676 & 3686517.50 & 3078.300 & 1.406 \\
SD & 0.001 & 835.228 & 0.504 & 0.001 \\
\%RSD & 0.084 & 0.023 & 0.016 & 0.037 \\
\hline
\end{tabular}

Name of drug-amlodipine

\begin{tabular}{lccccc}
\hline Injection No. & Retention time & Area & Theoretical plates & Tailing factor & Resolution \\
\hline 1 & 5.035 & 1353496.00 & 2982.438 & 1.403 & 11.721 \\
2 & 5.016 & 1354432.00 & 2978.862 & 1.405 & 11.690 \\
3 & 5.005 & 1354197.00 & 2976.722 & 1.407 & 1.406 \\
4 & 4.997 & 1353220.00 & 2983.002 & 1.408 & 11.659 \\
5 & 4.989 & 1352243.00 & 2989.282 & 1.4091 & 11.617 \\
6 & 4.981 & 1351266.00 & 2995.562 & 1.406 & 11.592 \\
Average & 5.004 & 1353142.33 & 2984.311 & 0.002 & 0.047 \\
SD & 0.020 & 1203.005 & 6.983 & 0.155 & 0.404 \\
\hline \%SD & 0.390 & 0.089 & 0.234 & & 0.04 \\
\hline
\end{tabular}

Selectivity. Selectivity is the ability to assess the analyte in the presence of components that may be expected to be present. Typically these might include impurities, degraded products, matrix, etc. Standard solutions (100\%) containing both the drugs was injected first. Then drugs solution containing three placebo formulations were injected one after another. Figure1 showed that in the presence of excipients the retention time of atenolol and amlodipine remain same. On the other hand no other peaks were found within 10 min run time of the chromatogram which proves the selectivity of the method.
Linearity. Linearity of the method was evaluated from the correlation coefficient of calibration curves that were constructed from average peak area of atenolol and amlodipine at different concentrations level $(80 \%, 90 \%, 100 \%, 110 \%$, and $120 \%)$. Correlation coefficient was 0.999 both for atenolol and amlodipine which prove that the method is linear for both atenolol and amlodipine. It means that the response is directly proportional to the concentration of analytes.

Accuracy. Accuracy is generally assessed by analyzing a sample with known concentration and comparing the measured value with the true value. The measured value was obtained by recovery test. 
Spiked amount of both atenolol and amlodipine were plotted against the recovery amount (Figure 2 and 3). The Correlation coefficient was 0.999 both for atenolol and amlodipine. In case of atenolol \% recovery was $99.51 \%-101.34 \%$ (average 100.72 , \% RSD 1.03) and in case of amlodipine \% recovery was $98.86 \%-100.14 \%$ (average $99.44 \%$ RSD 0.648). All the results indicate that the method is highly accurate.

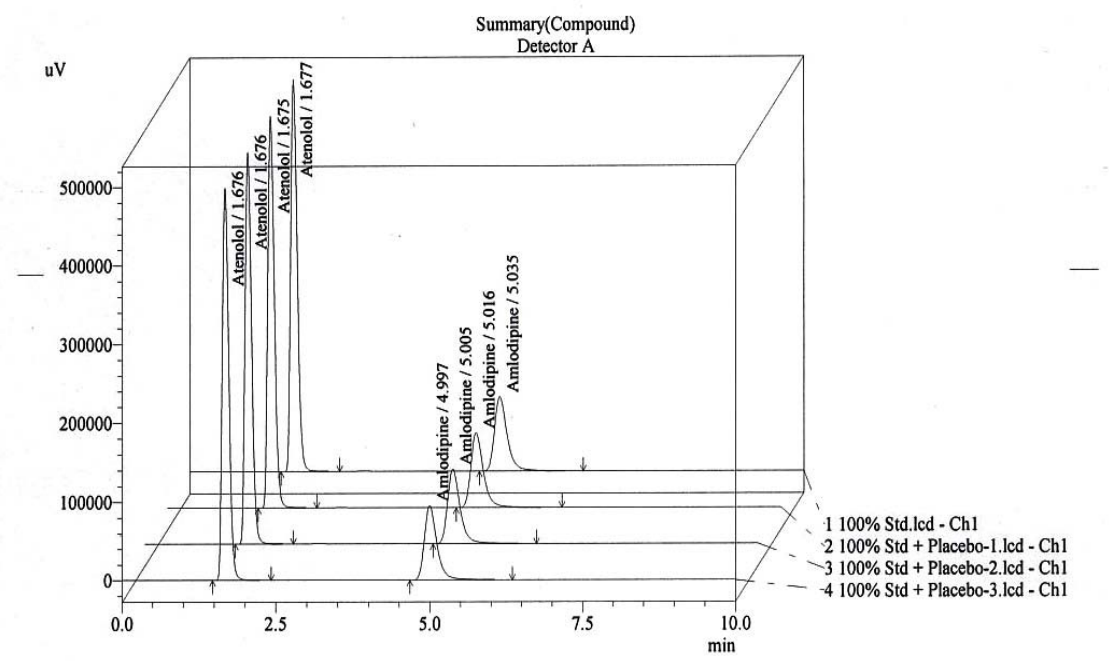

Figure 1. Chromatogram of atenolol and amlodipine along with 3 placebo formulations.

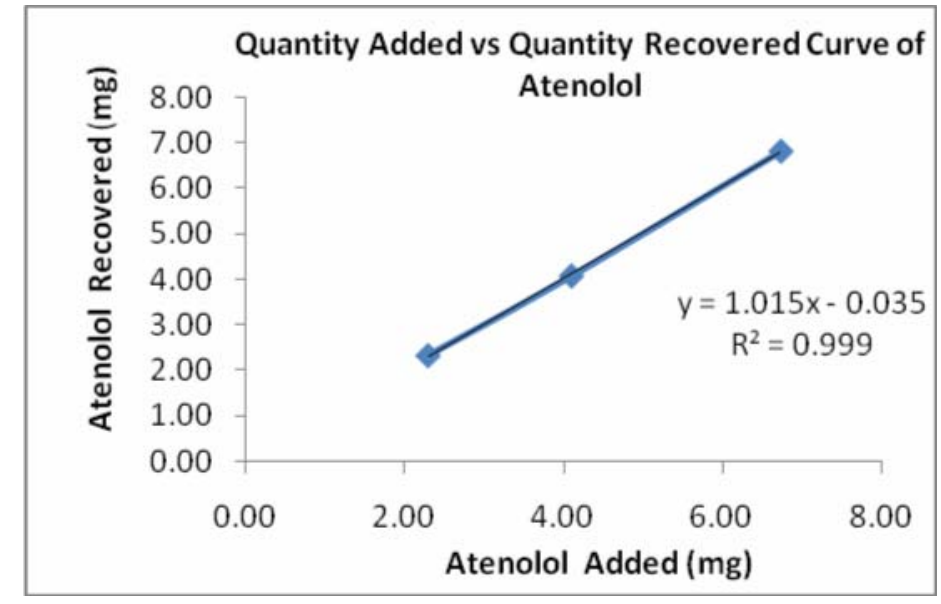

Figure 2. Accuracy curve of atenolol

Precision. The precision of an analytical method is the degree of agreement among individual test results when the method is applied repeatedly to multiple samplings of a homogeneous mixture. The precision of an analytical method is usually expressed as the standard deviation or relative standard deviation (coefficient of variation) of a series of measurements. Precision was measured by repeatability and intermediate precision.

Repeatability. Repeatability expresses the precision under the same operating conditions over a short interval of time. It is also termed intra-assay precision. Repeatability is usually demonstrated by repeated measurements of a single sample. Minimum 
four determinations at each of three concentrations across the intended range, or a minimum of six determinations at the test concentration are recommended.

The measurements for repeatability were done from $9.00 \mathrm{am}$ to $9.00 \mathrm{pm}$. Four determinations of three concentrations across the intended range (90\%, 100\% and $110 \%$ of target concentration) were included in the study. \% RSD of peak areas was calculated for various run. The method is highly precise as \% RSD of peak area was 0.053-0.152 in case of atenolol and 0.076-1.518 in case of amlodipine.

Intermediate precision. Intermediate precision is usually demonstrated by repeated measurements of the sample used in the repeatability experiment within the same laboratory. Usually the repeatability experiment is repeated on the same sample by a different analyst, on a different day, using different equipment if possible.

The data of table 2 and 3 showed the average results of intermediate precision of atenolol and amlodipine. The same concentration levels as in the repeatability experiment were used in this study. The results are obtained by 3 concentrations with 4 runs over 3 days. The average peak area obtained at different levels and different days indicate that the method is precise. Maximum \% RSD is 0.151 in case of atenolol and 1.51 in case of amlodipine.

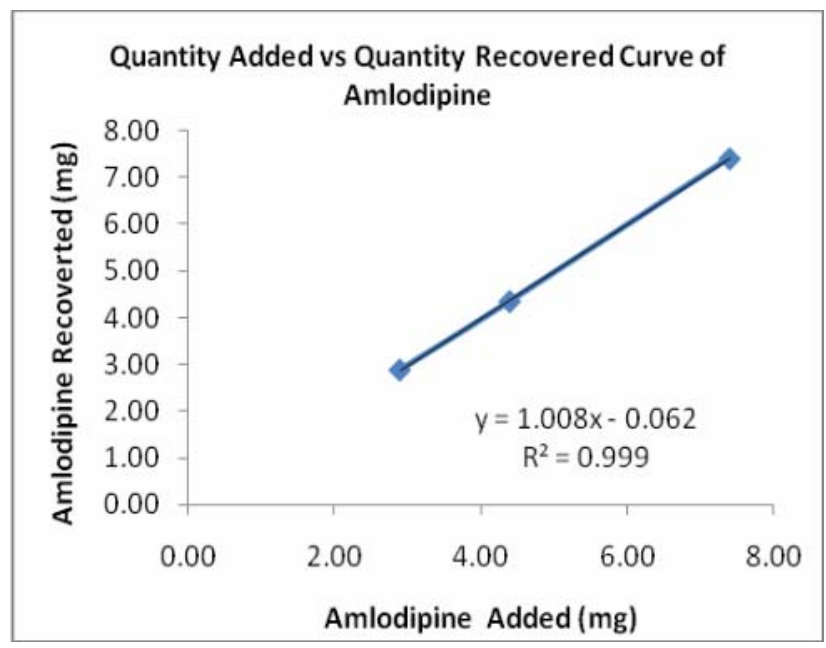

Figure 3. Accuracy curve of amlodipine

Table 2. Inter-day variability (three different concentrations of standard solution of atenolol, injected on different days).

\begin{tabular}{cccc}
\hline Days & Std conc. & Mean peak area of atenolol $(\mathrm{n}=4)$ & \% RSD \\
\hline \multirow{3}{*}{ 1st } & $90 \%$ & 3311515.25 & 0.0797 \\
& $100 \%$ & 3686255.75 & 0.0243 \\
\multirow{3}{*}{ 2nd } & $110 \%$ & 4031586.50 & 0.1105 \\
& $90 \%$ & 3312999.75 & 0.0529 \\
& $100 \%$ & 3686025.25 & 0.1518 \\
\multirow{3}{*}{ 3rd } & $110 \%$ & 4033851.00 & 0.0854 \\
& $90 \%$ & 3332855.75 & 0.0767 \\
& $100 \%$ & 3706295.75 & 0.0556 \\
\hline
\end{tabular}

Robustness. The robustness of a method is its ability to remain unaffected by small changes. Robustness study was performed by making slight variations in flow rate, amount of acetonitrile, and temperature. $100 \%$ atenolol and amlodipine sample solution was used in this study. The results of robustness in the 
present method showed no significant changes occurring over changes which are summarized in
Table 4. As the changes are not significant we can say that the method is robust.

Table 3. Inter-day variability (three different concentrations of standard solution of amlodipine injected on different days)

\begin{tabular}{lccc}
\hline Days & Std conc. & Mean peak area of amlodipine $(\mathrm{n}=4)$ & \% RSD \\
\hline \multirow{3}{*}{ 1st } & $90 \%$ & 1299744.00 & 0.1667 \\
& $100 \%$ & 1353835.75 & 0.0422 \\
\multirow{3}{*}{ 2nd } & $110 \%$ & 1613112.00 & 0.1691 \\
& $90 \%$ & 1300537.00 & 0.076 \\
& $100 \%$ & 1377701.25 & 1.5183 \\
\multirow{3}{*}{ 3rd } & $110 \%$ & 1616511.00 & 0.2024 \\
& $90 \%$ & 1315026.75 & 0.1273 \\
& $100 \%$ & 1368271.75 & 0.0341 \\
& $110 \%$ & 1633389.75 & 0.1308 \\
\hline
\end{tabular}

Table 4. Results for robustness test of atenolol and amlodipine

\begin{tabular}{ccccc}
\hline Parameters & Changes & $\begin{array}{c}\text { \% Recovery of } \\
\text { atenolol }\end{array}$ & $\begin{array}{c}\text { \% Recovery of } \\
\text { amlodipine }\end{array}$ & \% Target \\
\hline Flow Rate (ml/min) & 0.9 & 99.7 & 99.3 & 100 \\
Column Temperature & 1.1 & 99.6 & 99.4 & 100 \\
$\left({ }^{0} \mathrm{C}\right)$ & 28 & 99.6 & 99.5 & 100 \\
Acetonitrile Variation & 30 & 99.5 & 99.5 & 100 \\
& $10 \%$ & 99.6 & 99.7 & 100 \\
\hline
\end{tabular}

\section{Analysis of market products}

The proposed method was used to determine the potency of commercially available tablets (Six brands) containing $50 \mathrm{mg}$ of atenolol and $5 \mathrm{mg}$ of amlodipine. Three replicate determinations $(n=3)$ were carried out and the results are summarized in Table 5.

Table 5. Determination of atenolol and amlodipine in commercial formulations by high performance liquid chromatography

\begin{tabular}{ccccccc}
\hline \multirow{2}{*}{ Brand } & \multicolumn{3}{c}{ Atenolol } & \multicolumn{3}{c}{ Amlodipine } \\
\cline { 2 - 6 } & $\begin{array}{c}\text { Labeled amount } \\
(\mathrm{mg})\end{array}$ & $\begin{array}{c}\text { Observed } \\
\text { amount (mg) }\end{array}$ & Purity & $\begin{array}{c}\text { Labeled } \\
\text { amount (mg) }\end{array}$ & $\begin{array}{c}\text { Observed } \\
\text { amount (mg) }\end{array}$ & Purity \\
\hline T-1 & 50.00 & 50.01 & 100.02 & 5.00 & 4.98 & 99.60 \\
T-2 & 50.00 & 49.51 & 99.02 & 5.00 & 5.01 & 100.20 \\
T-3 & 50.00 & 49.54 & 99.08 & 5.00 & 4.98 & 99.60 \\
T-4 & 50.00 & 49.57 & 99.14 & 5.00 & 4.97 & 99.40 \\
T-5 & 50.00 & 49.37 & 98.74 & 5.00 & 5.02 & 100.40 \\
T-6 & 50.00 & 49.44 & 98.88 & 5.00 & 4.87 & 97.40 \\
\hline
\end{tabular}

\section{CONCLUSION}

The proposed high-performance liquid chromatographic method has been evaluated over the accuracy, precision and linearity and proved to be more convenient and effective for the quality control and identity of atenolol and amlodipine in pharmaceutical dosage forms. The measured signals were shown to be precise, accurate and linear over the concentration range tested (80-120\% of target concentration) with a correlation coefficient better than 0.999. Moreover, the lower solvent consumption along with the short analytical run time of 10 minutes leads to an environmentally friendly chromatographic procedure that allows the analysis of a large number of samples in a short period of time. Therefore, this HPLC method can be used as a routine sample 
analysis. Additionally in this method, there was no interference from matrix sources.

\section{REFERENCES}

1. Brunton, L.L. and Parker, K.L. 1996. Editors. Goodman \& Gilman's Manual of Pharmacology and Therapeutics. New York: McGraw Hill.

2. Tripathi K.D. 2003. Essential of Medical Pharmacology, 5th Ed., Jaypee Brothers Medical Publishers (P) Ltd., New Delhi

3. Williams A. 2004. Foye's Principles of Medicinal Chemistry, 5th Ed., published by B.I. publications Pvt. Ltd.

4. Prisant, L.M. 2002. Fixed low-dose combination in firstline treatment of hypertension. J. Hypertens. 20, S11-S19.

5. Li-Ping, X., Fu-Ming, S.H., Chao-Yu, M., Yúan-Ying, J. and Ding-Feng, S. (2005). Synergism of atenolol and amlodipine on lowering and stabilizing blood pressure in spontaneously hypertensive rat. Acta. Pharmacologica. Sinica, 26, 13031308.

6. Indian Pharmacopoeia, Vol. II., Published by the Controller of Publication Delhi, 1996.

7. British Pharmacopoeia, Vol. I., 2010. Published by MHRA, UK.

8. United States Pharmacopeia, 2009. Asian edition, US pharmacopoeial convention, Inc.: US; 2009.

9. European pharmacopoeia, 6th Ed. V-2, Council of Europe, 2008.

10. Johannsson M. 1988 Determination of atenolol in plasma by dual-column liquid chromatography and Fluorimetric, $J$. Chromatogr. 18, 265-272.

11 Patki, R.V., Tamhanker, C.P. and Tipnis, H.P.1994. Simple and rapid high performance liquid chromatographic estimation of amlodipine in pharmaceutical dosage forms. Indian Drugs 31, 560

12. Yeung, P.K., Mosher, S.J. and Pollack, P.T. 1991. High performance liquid chromatography assay for amlodipine: chemical stability and pharmacokinetics in rabbits. J Pharm Biomed Anal. 9, 565.

13. Josefsson, M., Zackrisson, A.L. and Norlander, B. 1995. Sensitive high performance liquid chromatographic analysis of amlodipine in human plasma with amperometric detection and a single step solid phase sample preparation. $J$ Chromatogr B Biomed Appl. 672, 310.
14. Bresford, A.P., Marcrac, P.V. and Stopher, D.A. 1987. Analysis of amlodipine in human plasma by gas chromatography. J Chromatogr. 420, 178.

15. Naidu, K.R., Kale, U.N. and Shingare, M.S. 2005. Stability indicating RP-HPLC method for simultaneous determination of Amlodipine and Benazepril $\mathrm{HCl}$ from their combination drug product, J. Pharm. Biomed. Anal. 39, 147-155.

16. Topale P.R., 2003. Simultaneous UV Spectrophotometric estimation of Amlodipine and Losartan Potassium in tablet dosage forms. Indian drugs. 40, 119-121.

17. Prasad, C.V.N., Saha R.N. and Parimoo P. 1999. Simultaneous determination of Amlodipine- Enalapril Maleate and Amlodipine-Lisinopril in combined tablet preparations by derivative Spectrophotometry, Pharmacy and Pharmacology communications. 5, 383-388.

18. El-Gindy A. 2005. HPLC and chemo metric-assisted spectrophotometric methods for simultaneous determination of Atenolol, amiloride hydrochloride and chlorthalidone, Farmaco. 60, 269-278.

19. Kasture, A.V. and Ramteke, M. 2006. Simultaneous UV Spectrophotometric method for the estimation of atenolol and amlodipine besylate in combined dosage form. Indian J. Pharm. Sci. 68, 394396.

20. Ravishankar, S., Nanjan, M.J., Vasudevan, M., Shaat, N. and Suresh, B. 1997. Simultaneous estimation of atenolol and amlodipine in formulations by reversed phase-HPLC. Indian J. Pharm. Sci. 59, 171-173.

21. Ranjan, k.B, Anwar U.I., Ahmed, M., Imam, B.W., Robiul, I., Alam, k., Belal, M.H. and Bytul, M.R. 2007. Simultaneous high-performance liquid chromatographic determination of atenolol and amlodipine in pharmaceutical-dosage form. Pak. J. Pharm. Sci. 20, 274-279

22. Argekar, A.P. and Powar S.G. 2000. Simultaneous determination of Atenolol and Amlodipine in tablet by high performance thin liquid chromatography. J Biomed. Anal. 21: 1137-42.

23. International Conference on Harmonisation. Draft Guideline on Validation of Analytical Procedures: 1995. Definitions and Terminology, Federal Register, 60, 11260.

24. Global Quality Guideline. Validation of Analytical Procedures. 2002; Number: G-6.9, Version: 1.0. 